\section{Achromatic surface color and the direction of illumination*}

\author{
HOWARD R, FLOCK $\dagger$ \\ York University, Downsview, Ontario, Canada
}

Hochberg and Beck (1954) found that an objectively upright trapezoid, when illuminated from above, appeared darker if it was viewed monocularly and lighter if it was viewed binocularly. Illuminated from in front, the same trapezoid then appeared lighter under monocular and darker under binocular viewing. Since the target appeared slanted under monocular but upright under binocular viewing, these changes in apparent lightness could be attributed, wholly or in part, to the apparent angle of incidence of the illumination on the surface. In two experiments, when $8 \cdot \mathrm{min}$ periods of dark adaptation were introduced between monocular and binocular viewing, but when the arrangements were otherwise approximately the same as those of Hochberg and Beck, their results could not be observed. A third experiment demonstrated that the monocularly observed trapezoids did appear slanted.

Results of some recent experiments can be interpreted to prove and others to disprove the hypothesis that apparent lightness (grayness) of a surface depends in part on perceiving or registering the direction of the illumination (Beck, 1965; Epstein, 1961; Flock \& Freedberg, 1970; Hochberg \& Beck, 1954). In fact, with one exception, these experiments are ambiguous as they apply to the issue. The one exception is an experiment by Hochberg and Beck (1954). That experiment, in the view of this writer, does seem to support the proposition that perception of illumination functions as a cue to apparent achromatic surface color (lightness).

Hochberg and Beck (1954) presented an upright trapezoid on a flat black bench. Surrounding the target were piles of blocks. The attached and cast shadows on the blocks presumably gave cues to the objective location and intensity of the light-source in the experimental space. Ss observed the target from $4 \mathrm{ft}$ in front and from approximately $2 \mathrm{ft}$ above it. If $S$ viewed the target with one eye, it appeared to lie flat on the bench, although it was objectively upright. Viewed with two eyes it appeared upright. In this way apparent angle of incidence of light on the surface was varied without changing the luminance of the surface, without changing the apparent or objective location and intensity of the source, and without changing the objective angle of incidence. In every case, for three different objective locations of the source (above, in front,

\footnotetext{
* This research was supported by the National Research Council of Canada Cirant No. APA-143. The experiments described here were carried out in early 1968.

Address: Department of Psychology, York University, Downsview, Ontario, Canada.
}

and to the side of the trapezoid), the Ss reported the surface to be a lighter gray when the apparent angle of incidence was larger, and a darker gray when the apparent angle of incidence was smaller; they reported no changes when the angles of incidence were the same. (Angle of incidence is defined by the angle formed by the axis normal to a point on the surface and a line connecting that point and the light source.) Accordingly, a hypothesis that fits their data (and their account of how a cue theorist would explain their data) is that: a surface will be reported to be a lighter gray when the apparent angle of incidence becomes larger, all other conditions remaining constant. 1

What was so compelling about Hochberg and Beck's (1954) experiment was the change in ocular combinations that produced a larger apparent angle of incidence and therefore a lightening of the target for the different loci of the illuminant. When the illuminant was above and slightly in front of the target, the apparent angle of incidence was increased by shifting from monocular to binocular viewing. The target, correspondingly, was reported to lighten from monocular to binocular viewing. When the illuminant was directly in front of the target, however, this order was reversed. Under this front illumination the apparent angle of incidence was increased by shifting from monocular to binocular viewing, and the target was reported lighter under binocular rather than under monocular viewing. Hence, as the illuminant was moved from above to in front, there was a shift in the ocular combinations that produced the lightening of the target. This state of affairs will be referred to hereafter as the ocular-shift effect and its presence will mean that the results corresponded to those observed by Hochberg and Beck. In demonstrating the presence of the ocular-shift effect, a comparison is first made between monocular and binocular judgments with the illuminant in a particular location; then the sign of the difference is shown to alter appropriately for the change in location of the illuminant.

Since Hochberg and Beck's study, no investigator has even attempted to show this ocular-shift effect under their experimental conditions. Instead, investigators have attempted to repeat parts of the original study, with results that have been ambiguous, I believe, as they have applied to the cue functions of perceived illumination. For example, Beck (1965, Study II) used substantially the same arrangements as did Hochberg and Beck, but with the source always overhead. The trapezoid was placed at four different distances from the $O$. For one of those distances (Position IV), the angle of incidence was, presumably, the same for both monocular and binocular viewing. As a result, judgments of lightness should not have changed, and did not change, from binocular to monocular viewing. For Beck's Position I, the illuminant was objectively behind the target and would presumably appear there under binocular viewing. But the target, nevertheless, had a luminance of approximately $1.3 \mathrm{fL}$. Hence the apparent angle of incidence was indeteruinate for the situation. In accordance with cue theory the $S$ would hold some unknown hypotheses about how the surface was being illuminated, and these hypotheses would in part determine his binocular judgments of the lightness of the surface. Under monocular viewing, however, when the target appeared flat, the angle of incidence would be determinant, although unspecifiable from Beck's description, and the $S$ might be expected to hold a different set of hypotheses. It is not clear, therefore, for such a complex situation what hypotheses the investigator should have held. For Beck's other two distances (Positions II and III), the description does not specify the objective angles of incidence, and the procedures did not measure the apparent slant of the target, from which apparent angles of incidence could have been presumed. Also unclear, therefore, would be the investigator's expectations. The same difficulties are to be found in Beck's (1965) Study I.

Most recently, Flock and Freedberg (1970) also replicated Hochberg and Beck, but used only overhead illumination. In two studies they found that the target monocularly appeared darker and that the magnitude of the effect increased as cues to the direction of the illuminant were 


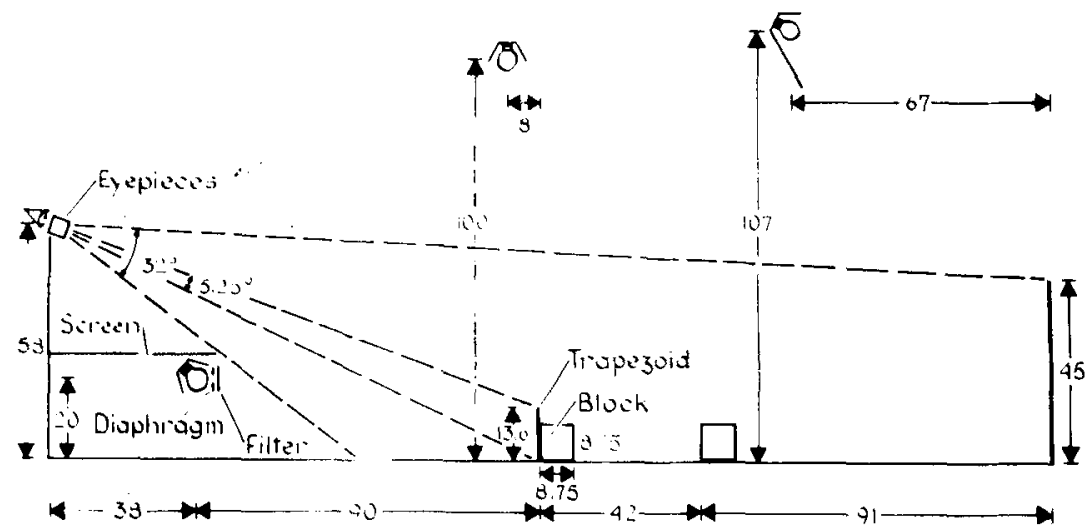

Fig. 1. Side view of layout (measurements in centimeters).

increased, as would be predicted from Hochberg and Beck's results. However, the magnitude of the effect was small, even when the cues were maximized. The magnitude of the largest effect implied that the target had monocularly slanted back 4.9 deg. For comparable conditions various investigators of the monocular slant of trapezoids (Dunn, 1966; Flock, 1965, 1967; Gogel, 1965; Smith, 1967) have reported angles of slant markedly in excess of $4.9 \mathrm{deg}$. It would seem, therefore, that the magnitude of the effect was too small to imply that the apparent direction of the illumination was wholly determining the perception of surface color. The effect could imply, however, that the apparent direction of the illumination was contributing in part to apparent surface color. Nevertheless, despite that possibility, Flock and Freedberg favored an alternative explanation.

Epstein (1961) has been the only investigator to attempt to replicate Hochberg and Beck with the illuminant in front. But Epstein also made no attempt to test for the ocular shift. His $\mathrm{S}$ s reported the target unchanged from monocular to binocular viewing. In his experiment, however, he may not have supplied cues to the direction of the source. Without those directional cues, Hochberg and Beck (1954) also found little or no difference from monocular to binocular viewing.

That is where the issue is now, as it has been recently investigated. It is difficult, if not dubious, to try to measure with any degree of accuracy the apparent slant of the target while $S$ is observing its lightness. Hence, the expected magnitude of the effect for the illuminant in a particular location generally depends on an assumed, but unmeasured, apparent slant of a monocularly viewed trapezoid. It is true, of course, that with the illuminant overhead and somewhat in front of the target, monocular darkening of the target (or binocular lightening) could be interpreted to mean that apparent angle of incidence had contributed to judged surface color. This would be more persuasive if, at the same time, it was shown that the target appeared binocularly darker and monocularly lighter when the illuminant was moved to the front.

In two of the three experiments described below, therefore, an attempt will be made to observe Hochberg and Beck's optical-shift effect. Experiments 1 and 2 are straightforward replications of Hochberg and Beck (1954), but with at least two major changes. Four targets are used instead of one, and, between monocular and binocular viewing, 8-min periods of dark-adaptation are introduced. In the first experiment each eye is used equally often, whereas in the second experiment all monocular viewing is with the same eye, which is, presumably, how Hochberg and Beck performed their experiment. The third experiment is concerned with whether the trapezoids did slant during monocular viewing.

\section{EXPERIMENT 1}

\section{Purpose}

To determine whether the optical-shift occurs when each eye is used equally often, when dark adaptation is introduced between monocular and binocular viewing, but when the arrangements are otherwise like those used by Hochberg and Beck.

\section{Subjects}

Four women and 12 men, undergraduates at York University, with a median age of 19 and naive to the purposes of the experiment, participated. All had $20 / 20$ uncorrected vision in each eye.

\section{Apparatus}

Four trapezoids were cut from Plexiglas so that the longer lower bases were $21.0 \mathrm{~cm}$, the shorter upper bases, $17.0 \mathrm{~cm}$, and the heights, $13.6 \mathrm{~cm}$. Each was covered with a different neutral (N) Munsell paper, 4.5, 5.5, 6.5, and 7.5. Double-sided tape was used to get a flat adhesion of the paper. Each of these trapezoids could be placed upright in the same position on the midline of a table, facing one of the ends of the table. Mounted to the back of each trapezoid was a small Plexiglas block with two guide holes that engaged a plate with two pins attached to the surface of the table. In this way each trapezoid could be quickly interchanged. The table, $250 \mathrm{~cm}$ long and $106 \mathrm{~cm}$ wide, was covered with black velvet, as was a wall that was $133 \mathrm{~cm}$ behind the position of the trapezoid. Except for the front end of the table, the other three sides were surrounded by heavy black drapes.

A pair of wooden blocks, $10 \mathrm{~cm}$ wide, $8.75 \mathrm{~cm}$ high, and $8.75 \mathrm{~cm}$ deep, was placed to either side of the upright trapezoid, separated from the end of its lower base by $5 \mathrm{~cm}$. Farther back on the table, $42 \mathrm{~cm}$ from the trapezoid and $16 \mathrm{~cm}$ to either side of the midline, a second pair of wooden blocks was placed. The highlights, cast, and attached shadows on the four blocks provided the cues to the location of the light source. Gradients of light on the black velvet background may have served the same function, if they were noticed.

A black card, $45 \mathrm{~cm}$ high and $61 \mathrm{~cm}$ wide, was vertically mounted on the back wall. In the center of the card a comparison (CO) series of 17 Munsell patches, from 1.5 to 9.5 , was arranged in two columns, Munsells 2.5 on the left and 9.6 on the right, with Munsell 5.5 placed between the two celumns at the bottom of the card, all mounted on their long axes. Munsells 1.5 and 9.5 were placed between the two columns at the top of the card, mounted on their short axes. Each patch was $8 \times 4 \mathrm{~cm}$ and was separated by $1 \mathrm{~cm}$ (Munsells 5, 5.5, and 6 were separated by $1.5 \mathrm{~cm}$ ). The area covered by the patches was $27 \mathrm{~cm}$ wide and $34 \mathrm{~cm}$ high. White india-ink numbers from 1 to 17 were placed to the outside of each patch, starting with 1.5 and moving counterclockwise, to identify the patch. The distance from the table to the first edge of a patch was $6 \mathrm{~cm}$.

The filament of a hooded tensor lamp (tungsten 12-V single-filament GE1133) was placed $100 \mathrm{~cm}$ above the midpoint of the lower base of the trapezoid and $8 \mathrm{~cm}$ in front of $\mathrm{it}$. The filament of a second hooded tensor lamp was placed $90 \mathrm{~cm}$ in front of the trapezoid and $20 \mathrm{~cm}$ above the surface of the table. An iris diaphragm, $2 \mathrm{~cm}$ in diam, was placed $5 \mathrm{~cm}$ in front of the lamp and prevented the beam from falling on the CO series. The lamp was 


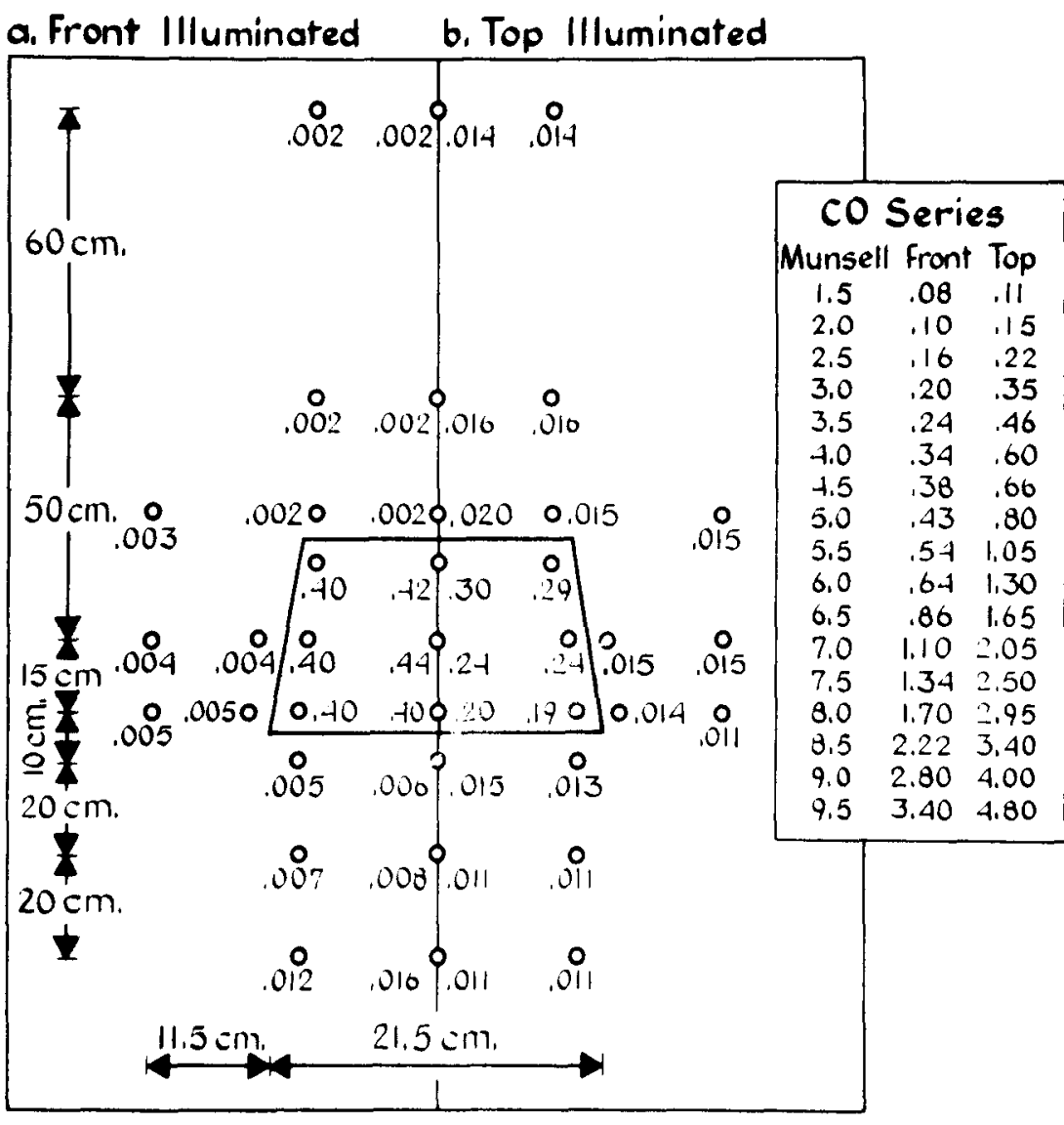

Fig. 2. Distribution of luminances (foot-lamberts) for scene, target, and $C O$ series without cubes.

angled slightly downward so that the shadow thrown by the trapezoid would fall just below the lowest $\mathrm{CO}$. A neutral density Wratten filter was placed in front of the lamp.

A third tensor lamp was mounted $107 \mathrm{~cm}$ above the table and $67 \mathrm{~cm}$ in front of the back wall, with the axis of the lamp angled to intersect the $9.5 \mathrm{CO}$ on the back wall. This lamp was suitably screened so that its light would fall on the $\mathrm{CO}$ series and any stray light would not change the luminance of the black background forward of $60 \mathrm{~cm}$ from the back wall when either the overhead or front sources were on. A control box allowed rapid switching from one source to a second.

Two eyepieces, each $6 \mathrm{~cm}$ in length with an inner diameter of $3.4 \mathrm{~cm}$, were mounted so that the viewing position would be $128 \mathrm{~cm}$ in front of the location of the trapezoids and $58 \mathrm{~cm}$ above the table. The eyepieces were individually adjustable and were converged inward and downward. Black disks could be introduced into either or both eyepieces to occlude the scene. A shutter placed to the inside of the eyepieces was operated by the $O$ to terminate or initiate a trial. A hydraulic chair positioned the $\mathrm{O}$ at the eyepieces.
With these arrangements as shown in Fig. 1, an $\mathrm{O}$ had a 32-deg field of view with either eye, had an unobstructed view with either eye of a trapezoid, the four blocks, the $\mathrm{CO}$ series, $40 \mathrm{~cm}$ of background in the midline in front of the target, almost the entire table behind the target, and $26 \mathrm{~cm}$ to one side and $50 \mathrm{~cm}$ to the other side of the midpoint of the lower trapezoidal base, depending on which eye was used. None of the three lamps was visible.

Figure 2 gives a representative sampling of the distribution of luminances (in foot-lamberts) over the background and over the Munsell 5.5 trapezoid for the top-illuminated (shown on the right side of Fig. 2) and front-illuminated (shown on the left side of Fig. 2) conditions. The inset in Fig. 2 gives the corresponding $\mathrm{CO}$ luminances.

The luminances in foot-lamberts of the $4.5,5.5,6.5,7.5 \mathrm{~N}$ Munsell trapezoids at their centers were: (1) top .17, .24, .40, and $.52:(2)$ front $-.30, .44, .65$, and .85 respectively. Although the luminances of the front-illuminated targets were slightly higher than for the top-illuminated targets, the background luminances were lower for the former, higher for the latter, as can be seen in Fig. 2. All luminance measurements were made with a Pritchard Spectraphotometer.

\section{Procedure}

Each S was told that he would see a four-sided figure (target) that would sometimes be a trapezoid, sometimes a rectangle, sometimes be upright, sometimes slanted back, and sometimes flat on a table. He was told to note the grayness of the figure and to select a $\mathrm{CO}$ that was the same gray. ${ }^{2}$ He was then told to check that his choice did indeed match the target. When he was satisfied with his choice, he activated the shutter. He was given several practice trials with monocular viewing. He was not asked to report the slant of the figure during the experiment. But during the practice trials all $S s$ reported that the monocularly observed trapezoids slanted back.

Each S made one judgment of the four trapezoids under the two illumination conditions and under monocular and binocular viewing. The experiment was then replicated, giving a total of 32 judgments. The experiment was divided into four blocks of eight judgments. Before the first block and between each block, $S$ was dark-adapted for $8 \mathrm{~min}$ in an almost totally dark room. Between each trial within a block he was dark-adapted for $30 \mathrm{sec}$.

Within each block the eight conditions of four Targets by two Illuminations were presented in a different random order. The four blocks represented a sequence of eye orders. The design of the experiment required a $S$ to use monocular and binocular viewing over the first two and last two blocks of judgments. An additional constraint required the $S$ to use a different eye for the two blocks of monocular conditions. These restrictions left eight possible eye orders, each of which was assigned to two Ss.

The Munsell judgments ${ }^{3}$ were transformed to log luminances (in footlamberts). The two monocular judgments were then averaged, as were the two binocular judgments, for each condition. This yielded eight monocular and eight binocular mean log judgments for each of the $16 \mathrm{Ss}$. The data were then analyzed using a three-factor by Ss design.

\section{Results}

The optical-shift effect did not occur. With the source overhead, binocular viewing did produce a lightening of the four trapezoids, a result observed by Hochberg and Beck. But the binocular lightening occurred also when the source was in front. This main effect of viewing was significant $(\mathrm{F}=21, \mathrm{df}=1 / 15, \mathrm{p}<.01)$ and is shown in Fig. 3, where each dashed line (monocular viewing) lies below the 


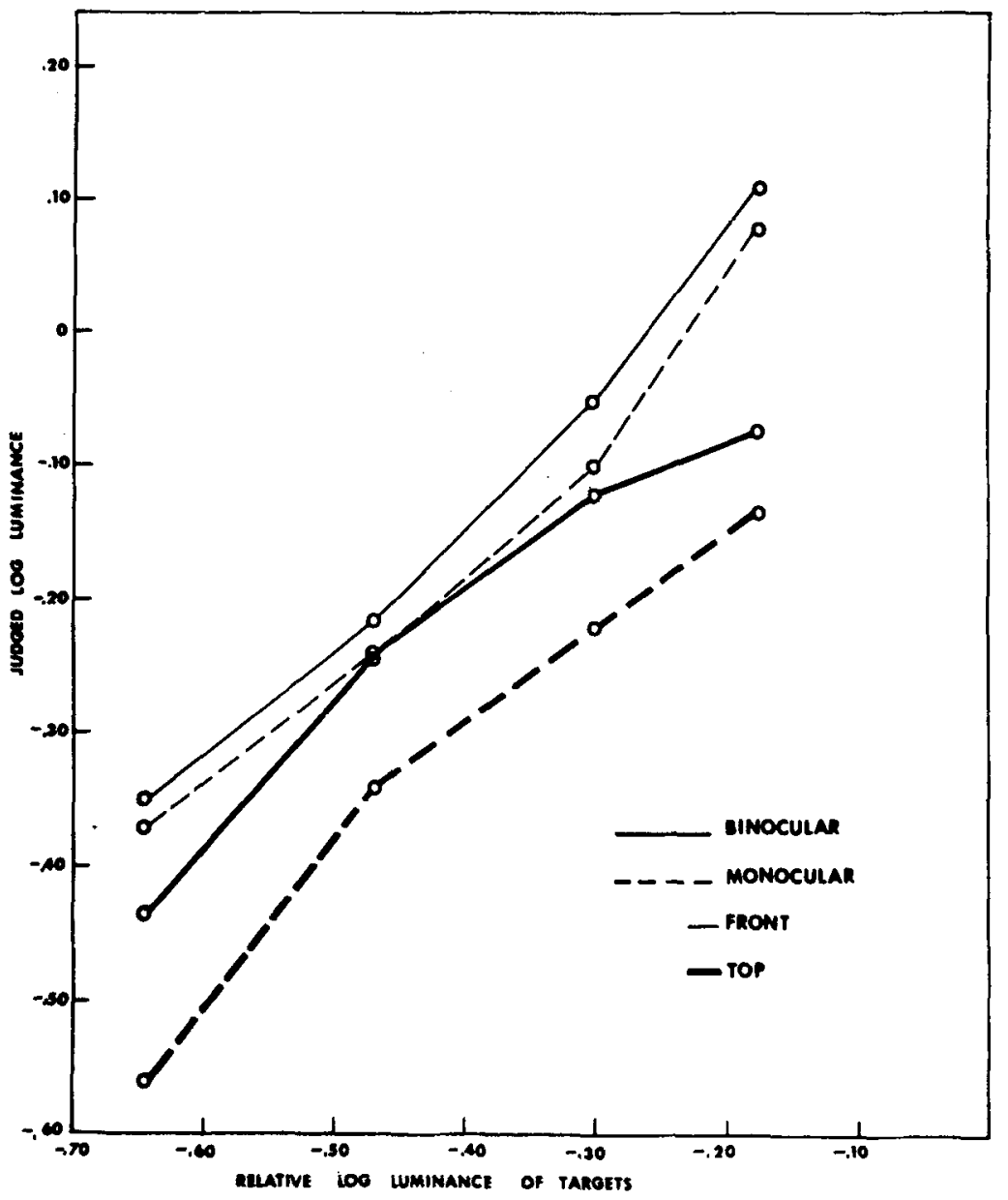

Fig. 3. Monocular and binocular judgments of four targets illuminated from above and in front.

appropriate solid line (binocular viewing). Not only did the optical-shift effect not occur in the mean data, but it was also not a frequent occurrence in the individual data. For each of the four targets there were $4,3,3$, and 2 , or a total of 12 shifts out of 64 ( 16 Ss by 4 targets) possible shifts. Of these 12,7 were contributed by three Ss. Hence, for nine Ss there were virtually no shifts at all.

The decrease in the lightness of the target from binocular to monocular viewing (V) was significantly less for front than for top illumination (E) ( $F$ of 4.94 for $\mathrm{V}$ by $\mathrm{E}, \mathrm{df}=1 / 15, \mathrm{p}<.05)$. This can be seen in Fig. 3, where the separation between the monocular and binocular curve is consistently smaller for front illumination and consistently larger for top illumination. The small, but consistent, effect between the monocular and binocular curves when illumination was in front is not dissimilar from Epstein's (1961) findings, in which there was a small, but insignificant, difference under comparable viewing conditions.
There were three other significant effects, but they had no direct bearing on the hypothesis being investigated. The significant effect of targets $(F=503$, $\mathrm{df}=3 / 45, \quad \mathrm{p}<.01)$ and of illumination $(F=35$, df $=1 / 15, \quad p<.01)$ would be expected because in the case of the former, lighter targets elicited lighter judgments, and in the case of the latter, since the front-illuminated targets were $.25 \mathrm{log} \mathrm{fL}$ higher in luminance, they correspondingly elicited lighter judgments. The third significant effect, Targets by Illumination, refers to the initial convergence then divergence of the curves, for top illumination, on the curves for front illumination ( $\mathrm{F}=14.4$, df $=3 / 45, \mathrm{p}<.01$ ).

\section{EXPERIMENT 2}

Hochberg and Beck's (1954) Ss presumably used the same monocular eye throughout their monocular observations. Hence, for the binocular observations the two eyes were differently dark adapted. In Experiment 1 each $S$ used each eye equally often. In the present experiment all

monocular observations were made with the same eye.

\section{Subjects}

Eight undergraduates, five men and three women, at York University, with a median age of 19 , and naive to the experiment, participated. All had uncorrected $20 / 20$ vision in each eye.

\section{Method}

The arrangements and instructions were exactly as they were in Experiment 1 except for one change in the procedure. Each of the eight possible eye orders was modified, substituting a left eye for a right eye in half of them and a right eye for a left eye in the other half. Hence, half of the Ss used the left eye and half the right eye throughout all monocular observations.

\section{Results}

The results of this experiment are shown in Fig. 4, where the mean $\log$ judgments are plotted against the relative $\log$ luminances of the targets. With one exception, the shape of the curves in Fig. 4 is approximately the same as in Fig. 3. The exception refers to the crossover of the dashed curve for the darkest target. Thus, when the source was overhead (bottom pair of curves), the Munsell 4.5 target appeared lighter when viewed binocularly, whereas when the source was in front (upper pair of curves), the same target appeared slightly darker when viewed binocularly.

\section{EXPERIMENT 3}

An objection might be raised to the procedure of not having instructed the Ss to see the target as flat before making each of their monocular judgments. Perhaps the results differed from those of Hochberg and Beck (1954) because monocularly as well as binocularly the target was upright.

There were several reasons for not instructing the $S$ s to see the target as flat before making each of their monocular judgments. First, a test of the shift did not depend on the target monocularly lying flat. So long as the target monocularly slanted back at all with top illumination or slanted back more than $16 \mathrm{deg}$ with front illumination, ${ }^{4}$ the appropriate slant conditions existed for observing the shift. Second, even if a naive $\mathrm{S}$ did, under instructions, get the target to lie flat, it might not stay there while it was inspected for its lightness. Third, the best assurance that, on the average, the target would monocularly appear to slant back and binocularly would appear upright was to arrange the conditions so that those slant phenomena would occur.

The experiment described below tested 


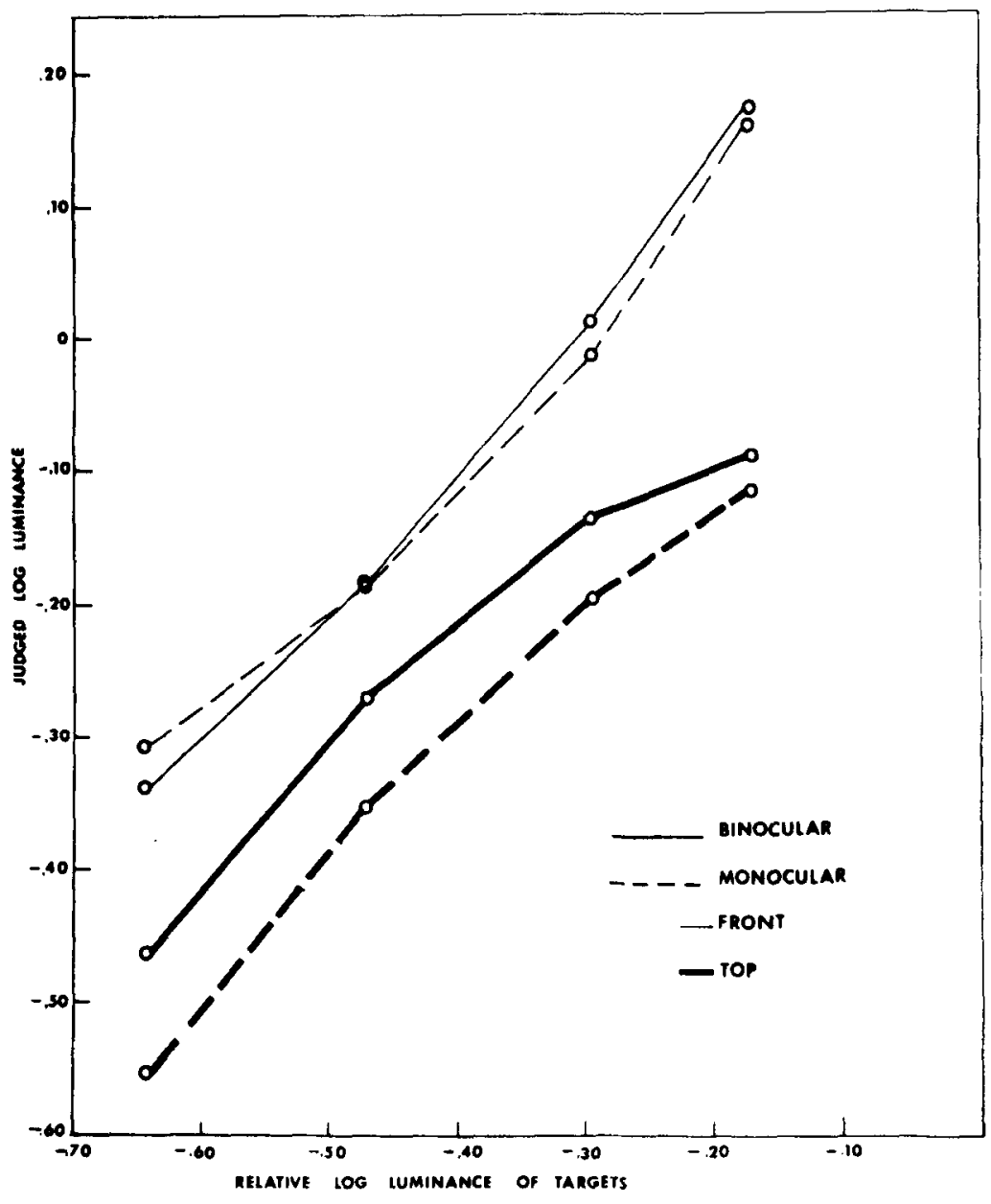

Fig. 4. Monocular and binocular judgments of four targets under two illuminations.

whether or not the stimulus conditions used in Experiments 1 and 2 would, on the average, monocularly elicit slant responses but binocularly elicit upright responses.

\section{Subjects}

Twelve undergraduates, seven men and five women, at York University, ages 19 to 24, naive to the purposes of the experiment, participated. All had 20/20 uncorrected vision in each eye.

\section{Method}

The arrangements were the same as in Experiment 1, except for some changes in the targets and the procedures. Three homogeneous trapezoids were made with $4: 0,5.5$, and $8.5 \mathrm{~N}$ Munsell surfaces but with the same sizes and proportions as before.

The Ss were asked to inspect the target and report its apparent position, whether slanting forward or upright, whether clearly slanting back (the Ss were shown a surface in full view at a $45-\mathrm{deg}$ slant), or whether lying flat on the table. They were then required to inspect the $\mathrm{CO}$ series for $5 \mathrm{sec}$ and again to give a judgment of the remaining nine Ss almost always (18 exceptions out of 108 responses) reported the surface as slanting back or lying flat.

Approximately half of the total of 50 exceptions occurred on the first judgment (24 responses) and half on the second judgment ( 26 responses) of a target. Also, 27 of the 50 exceptions occurred with overhead and 23 with front illumination.

Since $75 \%$ of the group almost always perceived the upright trapezoid to be slanted when viewed monocularly and upright when viewed binocularly, the assumptions made in Experiments 1 and 2 with regard to the apparent slant of the surface seem justified. Moreover, although $25 \%$ of the group reported the target to be mostly upright under monocular viewing, those Ss may have lapsed into reporting the objective situation, even though the trapezoid might have appeared to them to have some slant. In this context it should be remembered that Ss were instructed to report a target as slanted only if it appeared to have as much slant as a surface, in full binocular view, that was slanted $45 \mathrm{deg}$.

\section{SUMMARY}

The Hochberg and Beck optical-shift effect did not occur in two experiments, even though for $75 \%$ of the $S$ s the monocularly observed trapezoids could have been presumed to appear slanted and for nearly all of the Ss the binocularly observed trapezoids could have been presumed to appear upright. The arrangements in the two experiments were similar to those used by Hochberg and Beck, except for attempts to control for the differential sensitivity of the two eyes under the various conditions of viewing.

Hochberg and Beck's Ss alternated between one and two eyes, and presumably the same eye was used for all monocular observations. That meant that one of the binocular eyes was continuously being dark adapted during the monocular observations and that the two eyes were differentially sensitive during binocular observation. Although this asymmetry might have shifted the apparent lightness of the targets, it should also have shifted by the same amount the apparent lightness of the $\mathrm{CO}$ series and thereby have been cancelled out as a source of the effects. Nevertheless, the asymmetry was present in Hochberg and Beck's experiment, and it tended to be controlled in the present experiments. Whether or not that is the correct explanation for the difference in results, however, cannot be determined from these experiments because no attempt was made to repeat Hochberg and Beck under their conditions of alternating the monocular and binocular observations. But when eye 
usage was altered, as in Experiment 2, toward the procedure used by Hochberg and Beck, where the same eye was used in all monocular observations, the results did tend to change in the direction of Hochberg and Beck's results, although the change was too little to produce their optical-shift effect.

Whatever the ultimate explanation of the Hochberg and Beck shift, it would seem that it is not easily replicable and that there is at least some doubt that cues to the illumination play a part in it.

\section{REFERENCES}

BECK, J. Apparent spatial position and the perception of lightness. Journal of Experimental Psychology, 1965, 69, 170-179.

DUNN, B. E. Perceived slant as a function of direction of regard. Psychonomic Science, 1966, 4, 297-298.

EPSTEIN, W. Phenomenal orientation and perceived achromatic color. The Journal of Psychology, 1961, 52, 51-53.
FLOCK, H. R. Optical texture and linear perspective as stimuli for slant perception. Psychological Review, 1965, 72, 505-514.

FLOCK, H. R. Slant judgments of single rectangles at a slant. Psychonomic Science, $1967,7,57-58$.

FLOCK, H. R., \& FREEDBIERG, E. Perceived angle of incidence and achromatic surface color. Perception \& Psychophysics, 1970, 8, 251-256.

GULiLL, W. C. Equidistance tendency and its consequences. Psychological Bulletin, 1965, 64, 153-163.

HOCHBERG, J. E., \& BECK, J. Apparent spatial arrangements and perceived brightness. Journal of Experimental Psychology, 1954, 47, 263-266.

SMITH, A. H. Perceived slant as a function of stimulus contour and vertical dimension. Perceptual \& Motor Skills, 1967, 24, 167-173.

\section{NOTES}

1. Perception of illumination and its direction as a cue for perceptions of achromatic surface color should be distinguished from albedo theories, which may also depend on cues. Albedo theory is implied by 1'lock and Freedberg's
(1970) Eq. 2, or by their Eq. 3 with the assumption of an internalized standard illuminant. More general cue theories, with their expectations of relative changes in surface color as perceptions of the illumination are varied, are implied by Flock and Freedberg's Eq. 3 and are what will be discussed and tested in this paper.

2. Ss were encouraged in making their judgments to extrapolate as fincly as they wished between the 17 Munsell values composing the $\mathrm{CO}$ series.

3. The raw data for this experiment and for Experiment 2 below, as Tables A and B, may be obtained free of charge from the editorial office of this journal, Document No. P933.

4. It was necessary to elevate the front lamp $20 \mathrm{~cm}$ above the table so that the shadow of the trapezoid would not fall on the COs. This made the angle of incidence $8 \mathrm{deg}$ for the upright trapezoid. At a target slant of 8 deg the angle of incidence would be $0 \mathrm{deg}$. Only when the trapezoid slanted back more than 16 deg would the angle have become greater than 8 deg.

(Accepted for publication May 28, 1970.) 